\title{
Association of Glutathione-S-Transferase M1, P1, and T1 Gene Polymorphisms with Treatment Response in Childhood Acute Lymphocytic Leukemia
}

\author{
April Grace Dion-Berboso, ${ }^{1,2}$ Ma. Melanie Liberty B. Alcausin, ${ }^{1,2}$ \\ Carmencita D. Padilla, ${ }^{1,2}$ Pamela D. Fajardo ${ }^{1}$ and Catherine Lynn T. Silao ${ }^{1,2}$ \\ ${ }^{1}$ Department of Pediatrics, College of Medicine and Philippine General Hospital, University of the Philippines Manila \\ 2Institute of Human Genetics, National Institutes of Health Philippines, University of the Philippines Manila
}

\begin{abstract}
There is still a strong need for new treatment strategies that will maintain remission and prolong survival in patients with acute lymphoblastic leukemia (ALL). The glutathione-S-transferase (GST) enzymes, which are coded by highly polymorphic genes, have been associated with the risk of developing cancer and were found to regulate effect of cancer treatment drugs.
\end{abstract}

Objectives. The present study determines the association of GSTM1, GSTP1 and GSTT1 polymorphisms and treatment response in terms of occurrence of adverse events and relapse in ALL in Filipino children.

Methods. This is a follow up study on the 2007 investigation done by Alcausin et al. which determined the association of the GST P1, M1, and T1 polymorphisms and occurrence of ALL. Fouryear follow-up data were available for 46 out of the 50 patients from January 2007 to May 2011. Odds ratios (OR) as measures of association of GST M1, P1 and T1 gene polymorphisms with treatment outcomes were estimated at 95\% confidence interval.

Results. Results show a trend towards predisposition to elevation of liver enzymes in patients with GSTT1 and GSTP1 mutant genotypes showing an $\mathrm{OR}(95 \% \mathrm{Cl})$ of $3.0(0.29-31.2)$ and 3.83 $(0.37-40.0)$, respectively. There is also a trend towards development of infection in patients with the GSTT1 null genotype with an $\mathrm{OR}(95 \% \mathrm{Cl})$ of $2.0(0.62-6.49)$. The presence of GSTM1 null genotype showed a trend towards protection from occurrence of relapse basing on both crude and adjusted ORs, $0.58(0.16-2.07)$ and $0.23(0.05-1.20)$, respectively. However, these results are not statistically significant.

\footnotetext{
Presented as a poster at the 10th Asia-Pacific Conference on Human Genetics December 5-8, 2012, Crown Plaza Hotel, Kuala Lumpur, Malaysia

Corresponding author: April Grace Dion-Berboso, MD Institute of Human Genetics

National Institutes of Health

University of the Philippines Manila

623 Pedro Gil St., Ermita, Manila 1000 Philippines

Telephone: +6323760962

Email: agberboso@post.upm.edu.ph
}

Conclusion. The GSTP1 heterozygous genotype conferred increased predisposition to elevation of liver enzymes while the GSTT1 null genotype was shown to be a possible risk factor towards the occurrence of both infection and elevation of liver enzymes during chemotherapy. Furthermore, the GSTM1 null genotype appears to be protective from occurrence of relapse. It is recommended to do similar large-scale studies in the future to obtain more conclusive results.

Key Words: Glutathione-S-transferase, acute lymphocytic leukemia, gene polymorphisms, adverse events, relapse

\section{Introduction}

Acute lymphoblastic leukemia is predominantly a disease of childhood accounting for about $77 \%$ of all leukemia cases in children and approximately $25 \%$ of all childhood cancers. ${ }^{1}$ In the Pediatric Hematology Section of the Philippine General Hospital, an average of 58 new cases is diagnosed yearly. Analysis using the Surveillance, Epidemiology, and End Results (SEER) database showed a 10-year survival rate of $87.8 \%$ in girls and $83.6 \%$ in boys. Further improvement in survival rates are expected with continuous advances in treatment such as in targeted and personalized therapy. ${ }^{1}$

The disciplines of pharmacogenetics and pharmacogenomics are emerging strategies aiming to improve treatment outcomes by personalizing therapy based on an individual's genotype. Genetic information has been used in disease risk identification, treatment choice and drug dosing in different types of diseases particularly cancer. ${ }^{2}$ In leukemias, one treatment strategy for improving cure has been to modify the intensity of therapy based on acquired genetic characteristics. Acute lymphoblastic leukemia treatment involves combination chemotherapy, thus, if the genetic determinants of interpatient variability in drug pharmacodynamics are better known, tailoring drug therapy based on these factors may improve outcome. ${ }^{3}$ For most leukemias, multiple genetic polymorphisms of metabolizing enzymes may interact with environmental, dietary, maternal and other external factors to affect the development of ALL. Also, the genes that encode these drug-metabolizing enzymes can influence the efficacy and toxicity of 
chemotherapy. For example, polymorphisms in the gene for thiopurine methyltransferase, an enzyme that catalyzes Smethylation (inactivation) of mercaptopurine, render the protein susceptible to degradation. Hence, when treated with standard doses of mercaptopurine, the patients who carry at least one variant allele, have an increased risk of hematopoietic toxic effects and tend to have longer periods of leukemia-free survival than do patients with two wildtype alleles. ${ }^{4}$

Glutathione S-transferases (GSTs) are a family of cytosolic enzymes involved in the detoxifications of various exogenous and endogenous reactive species. GSTs function as dimers by catalyzing the conjugation of mutagenic electrophilic substances to glutathione which is water soluble and can easily be excreted from the body. ${ }^{5}$ The human GST genes are divided into four major subfamilies designated as GST $\alpha$ or A, GST $\mu$ or M, GST $\theta$ or T, and GST $\pi$ or $P$. The genes have been well characterized and known to be polymorphic, with different polymorphism frequencies by ethnicity. The percentage of individuals who do not express the GSTM1 enzyme due to a homozygous gene deletion is higher in Caucasians and Asians than in Africans. About $60 \%$ of Asians, $40 \%$ of Africans and $20 \%$ of Caucasians do not express the GSTT1 enzyme. These homozygous gene deletions, called null genotypes, are denoted as GSTM1*0/*0 and $G S T T 1^{*} 0{ }^{*} 0$. Polymorphisms of GSTM1, GSTT1, and GSTP1 have been shown to be associated with susceptibility to various forms of cancer, resistance to chemotherapy treatment, and disease outcomes. ${ }^{6}$ In a 2008 study by Rimando et al., on the prevalence of GSTT1, GSTM1 and NQ01 $(609 \mathrm{C}>\mathrm{T})$ in 60 Filipino children with ALL, it was shown that the GSTM1 null and NQO1 wild-type genotypes are independently associated with the risk of ALL in Filipino patients. $^{7}$

Environmental carcinogens are detoxified through the GST system. Inter-individual differences in GST enzyme activity mediated by polymorphic genes have been suggested to influence varying susceptibility to environmental cancer. In contrast, GST genotypes conferring lower enzyme activity may be of advantage to individuals undergoing chemotherapy for neoplastic disease since reduced detoxification potentially enhances effectiveness of cytotoxic drugs. In India, Haranatha and Kaiser's study showed that patients with double gene deletions of GSTT1 and GSTM1 had improved treatment outcome in childhood ALL. ${ }^{5}$ However, a study done by Stanulla et al. which involved several centers in Germany, Austria and Switzerland showed that polymorphisms within genes of the GST superfamily may be associated with risk of relapse in childhood ALL. ${ }^{8}$ Also, a study done by Imanishi et al. showed that GSTM1 positive/null polymorphism could be a predictor for methotrexate hepatotoxicity in acute lymphoblastic leukemia and lymphoma. ${ }^{9}$ A study by Suneetha et al. on the role of GST and CYP1A1*2A polymorphisms in the therapy outcome of South Indian ALL patients showed that GSTP1 (Val allele) either in heterozygous or homozygous condition was associated with poor outcome. ${ }^{10}$

A study done by Alcausin et al. on the association of genetic polymorphisms of Glutathione-S-transferase P1, M1, and $\mathrm{T} 1$ in Filipino pediatric ALL patients showed that the presence of the wild type genotype GSTT1 and GSTM1 polymorphism appear protective while having the GSTP1 wild type genotype confers increased risk for ALL. ${ }^{11}$ This present study made use of the same study sample of Alcausin et al. to determine the association of GSTM1, GSTP1 and GSTT1 polymorphisms and treatment response in terms of occurrence of adverse events and relapse in ALL in Filipino children.

This study is the first in the Philippines to investigate the association between GST polymorphisms and treatment response in terms of occurrence of adverse events and relapse. The results of the study will provide baseline data for larger investigations on the role of GST polymorphisms in childhood ALL treatment outcome for the purpose of developing better, safer, more efficacious, and individualized treatment strategies in the future.

\section{Methods}

\section{Subjects}

The study sample is comprised of 50 acute lymphocytic leukemia patients, 18 years of age and below, who were diagnosed with ALL through morphology and/or flow cytometry, and undergoing chemotherapy at the Department of Pediatrics, University of the PhilippinesPhilippine General Hospital from January 2007 to June 2007. The present study involved the same ALL sample included in the 2007 study done by Alcausin et al. entitled, Genetic polymorphisms of Glutathione-S-Transferases P1, T1 and M1 in Pediatric Patients with Acute Lymphocytic Leukemia in a Philippine Tertiary Hospital, which investigated the association of the GST P1, M1, and T1 polymorphisms and acute lymphocytic leukemia. ${ }^{11}$ This study is a continuation of the aforementioned matched case-control study.

\section{Genotyping}

The study done by Alcausin et al. involved genomic DNA extraction from peripheral blood and determination of GST M1 and T1 polymorphisms using polymerase chain reaction (PCR) and determination of GST P1 polymorphisms using PCR-Restriction Fragment Length Polymorphism (RFLP). ${ }^{11}$

\section{Data Collection}

A chart review of the study sample was done to gather information on demographic data, pertinent history, laboratory results, treatment received, adverse events and 
occurrence of relapse. Adverse events included nonspecific findings such as fever, infection, nausea, weakness, weight loss and organ involvement which may include hematologic (bleeding, thrombocytopenia, leucopenia, coagulation defects, chemotherapy-induced myelosuppression), gastrointestinal (stomatitis, vomiting, diarrhea, constipation), liver (jaundice, elevation in liver enzymes), renal (increase in creatinine, proteinuria, hematuria, electrolyte abnormalities), cardiac (decrease in ejection fraction, ischemia, pericardial effusion, hypotension, hypertension), pulmonary (pleural effusion, arterial blood gas abnormalities), and neurologic (mood changes, headache, hearing and vision problems, motor and sensory changes). Mortality during chemotherapy was handled as an adverse event.

Three (3) patients who had missing charts were excluded from the study. One (1) patient who died prior to commencement of treatment was likewise excluded from the study.

The study received ethics approval from the Ethics Review Board of the Philippine General Hospital.

\section{Data Analysis}

This is a cross sectional study that looked into the association of GST M1, P1, and T1 gene polymorphisms with treatment response in terms of occurrence of adverse events and relapse in a population of children with acute lymphocytic leukemia in the wards of the Philippine General Hospital. Odds ratios (OR) as measures of association of GST M1, P1 and T1 gene polymorphisms with treatment outcomes were estimated at $95 \%$ confidence interval. Where feasible, estimates were adjusted for age, gender, and presence of CNS disease at diagnosis using multiple logistic regression.

\section{Results}

\section{Demographics and Clinical Characteristics of the Study Sample}

Four-year follow-up data were available for 46 of the 50 patients from January 2007 to May 2011. The patients were regularly seen at the Hematology-Oncology Outpatient Clinic of the Department of Pediatrics. Forty-three (43) patients were treated using the Children's Cancer Group (CCG) protocol, 2 patients used the Standard High-Risk ALL protocol, and 1 patient used the Berlin-Frankfurt-Munich (BFM) protocol. There was a 1.6:1 male preponderance and $80 \%$ of the patients fell under the 1-9 year age group. Twenty-nine (29) patients had immunophenotyping done and $93 \%$ of whom had B lineage type of ALL. Of the 9 patients who had cytogenetic studies done, 3 had abnormal karyotypes. Twenty-two percent had a family history of malignancy and 13\% had CNS disease at diagnosis (Table 1).
Table 1. Demographic Characteristics of the Study Sample.

\begin{tabular}{lc}
\hline Gender & \\
Male & 28 \\
Female & 18 \\
Age at Diagnosis & \\
$\quad$ <1year & 0 \\
1-9 years & 37 \\
10-19 years & 9 \\
Immunophenotype/Lineage & \\
$\quad$ B-Lineage & 27 \\
T-Lineage & 2 \\
$\quad$ Unspecified & 17 \\
Cytogenetics & \\
$\quad$ Normal & 6 \\
Abnormal & 3 \\
Not done & 37 \\
Family History of Malignancy & \\
Present & 10 \\
Absent & 36 \\
CNS Disease at Diagnosis & \\
Present & \\
Absent & 6 \\
\hline
\end{tabular}

\section{GST Genotypes and Occurrence of Adverse Events}

Eighty-five percent of the patients developed adverse events. Adverse events occurred slightly less often in the GSTM1 mutant genotype $(84.4 \%$ of 32$)$ as compared to the GSTM1 wild genotype ( $85.7 \%$ of 14$)$ with an OR $(95 \% \mathrm{CI})$ of 0.9 (0.08-6.54). Adverse events occurred slightly less often in the GSTP1 mutant heterozygous genotype $(85.7 \%$ of 21$)$ as compared to the GSTP1 wild genotype (87.5\% of 24$)$ with an OR $(95 \% \mathrm{CI})$ of $0.86(0.15-4.8)$. The presence of GSTT1 null genotype showed a trend towards predisposition to adverse events showing an OR (95\% CI) of 1.6 (0.23-11.95), however, the association is not statistically significant. Also, this trend was weakened when adjusted odds ratios were computed controlling for sex and age at diagnosis in terms of occurrence of adverse events showing an OR (95\% CI) of 1.15 (0.20-6.69) (Table 2 and Table 5).

Table 2. Distribution of GSTM1, GSTT1, and GSTP1 Genotypes and their association with occurrence of adverse events.

\begin{tabular}{ccccc}
\hline GENOTYPE & $\begin{array}{c}\text { No } \\
\text { Adverse } \\
\text { Events }\end{array}$ & $\begin{array}{c}\text { Presence of } \\
\text { Adverse } \\
\text { Events }\end{array}$ & OR (95\% CI) & P value \\
\hline $\begin{array}{c}\text { GSTM1 } \\
\text { Present }\end{array}$ & $2(14.3 \%)$ & $12(85.7 \%)$ & & \\
Null & $5(15.6 \%)$ & $27(84.4 \%)$ & $0.9(0.08-6.54)$ & 0.91 \\
GSTT1 & & & & \\
Present & $4(18.2 \%)$ & $18(81.8 \%)$ & & \\
Null & $3(12.5 \%)$ & $21(87.5 \%)$ & $1.6(0.23-11.95)$ & 0.59 \\
GSTP1 & & & & 0.86 \\
Ile/Ile & $3(12.5 \%)$ & $21(87.5 \%)$ & & \\
Ile/Val & $3(14.3 \%)$ & $18(85.7 \%)$ & $0.86(0.15-4.8)$ & \\
Val/Val & $1(100 \%)$ & $0(0 \%)$ & & \\
\hline
\end{tabular}


Table 3. Distribution of GSTM1, GSTT1 and GSTP1 genotypes and their association with occurrence of specific adverse events.

\begin{tabular}{|c|c|c|c|c|c|c|c|c|c|}
\hline \multirow[b]{2}{*}{ Adverse Events (\%) } & \multicolumn{3}{|c|}{ GSTM1 } & \multicolumn{3}{|c|}{ GSTT1 } & \multicolumn{3}{|c|}{ GSTP1 } \\
\hline & Null & Present & OR $(95 \% \mathrm{CI})$ & Null & Present & OR $(95 \% \mathrm{CI})$ & Ile/Val & Ile/Ile & OR $(95 \% \mathrm{CI})$ \\
\hline \multicolumn{10}{|l|}{ Fever $(46 \%)$} \\
\hline Yes & 6 & 5 & 0.42 & 3 & 8 & 0.25 & 6 & 5 & 1.52 \\
\hline No & 26 & 9 & $(0.1-1.7)$ & 21 & 14 & $(0.06-1.1)$ & 15 & 19 & $(039-5.96)$ \\
\hline \multicolumn{10}{|l|}{ Infection (54\%) } \\
\hline Yes & 17 & 8 & 0.85 & 15 & 10 & 2.0 & 11 & 14 & 0.79 \\
\hline No & 15 & 6 & $(0.24-3.01)$ & 9 & 12 & $(0.62-6.49)$ & 10 & 10 & $(0.24-2.56)$ \\
\hline \multicolumn{10}{|l|}{ Vomiting (13\%) } \\
\hline Yes & 3 & 3 & 0.38 & 2 & 4 & 0.41 & 3 & 3 & 1.17 \\
\hline No & 29 & 11 & $(0.07-2.17)$ & 22 & 18 & $(0.07-2.49)$ & 18 & 21 & $(0.21-6.51)$ \\
\hline \multicolumn{10}{|c|}{ Elevation of liver enzymes (9\%) } \\
\hline Yes & 3 & 1 & 1.34 & 3 & 1 & 3.0 & 3 & 1 & 3.83 \\
\hline No & 29 & 13 & $(0.13-14.18)$ & 21 & 21 & $(0.29-31.2)$ & 18 & 23 & $(0.37-40.0)$ \\
\hline \multicolumn{10}{|c|}{ Allergy L-Asparaginase (Leunase) (13\%) } \\
\hline Yes & 5 & 1 & 2.41 & 2 & 4 & 0.41 & 2 & 4 & 0.53 \\
\hline No & 27 & 13 & $(0.25-22.8)$ & 22 & 18 & $(0.07-2.49)$ & 19 & 20 & $(0.09-3.22)$ \\
\hline \multicolumn{10}{|c|}{ Chemotherapy-induced myelosuppresion (33\%) } \\
\hline Yes & 9 & 6 & 1.6 & 9 & 6 & 1.6 & 7 & 8 & 1 \\
\hline No & 15 & 16 & $(0.46-5.59)$ & 15 & 16 & $(0.46-5.59)$ & 14 & 16 & $(0.29-3.46)$ \\
\hline \multicolumn{10}{|l|}{ Mortality (33\%) } \\
\hline Yes & 11 & 4 & 1.31 & 9 & 6 & 1.6 & 5 & 10 & 0.44 \\
\hline No & 21 & 10 & $(0.33-5.15)$ & 15 & 16 & $(0.46-5.59)$ & 16 & 14 & $(0.12-1.59)$ \\
\hline
\end{tabular}

The frequency of occurrence of specific adverse events and their association with GST polymorphisms was also investigated. The most common adverse event experienced by the study sample was infection at $54 \%$, followed by fever at $46 \%$ and chemotherapy-induced myelosuppression at $33 \%$. Allergy to L-Asparaginase (Leunase), vomiting, and elevation of liver enzymes occurred in 13\%, 13\% and $9 \%$ of the patients, respectively. Thirty-three percent of the patients died, $80 \%$ of whom had relapse as a cause of death. There is a trend observed towards predisposition to elevation of liver enzymes in patients with GSTT1 and GSTP1 mutant genotypes showing an OR (95\% CI) of 3.0 (0.29-31.2) and $3.83(0.37-40.0)$, respectively. There is also a trend towards development of infection in patients with the GSTT1 null genotype with an OR (95\% CI) of 2.0 (0.62- 6.49). However, these associations are not statistically significant (Table 3 ).

Forty-eight percent of the patients had relapse. Relapse occurred more often in the GSTT1 mutant genotype $(50.0 \%$ of 24$)$ as compared to the wild (45.4\% of 22$)$ with an OR (95\% CI) of 1.20 (0.38-3.83). Relapse occurred more often in the GSTP1 mutant heterozygous genotype (52.4\% of 21$)$ as compared to the wild ( $45.8 \%$ of 24$)$ with an OR $(95 \% \mathrm{CI})$ of 1.3 (0.40-4.21). The presence of GSTM1 null genotype showed a trend towards protection from occurrence of relapse basing on both crude and adjusted ORs, 0.58 (0.16$2.07)$ and 0.23 (0.05-1.20), respectively, however these results are also not statistically significant (Table 4 and Table 6).

Adjusted odds ratios were also computed controlling for sex and age at diagnosis in terms of occurrence of adverse events (Table 5) and controlling for sex, age at diagnosis, and presence of CNS disease at diagnosis in terms of occurrence of relapse (Table 6). The adjusted odds ratios showed almost similar results as compared to the crude odds ratios except in GSTT1 genotype wherein the adjusted OR for occurrence of relapse is decreased to 0.69 from a crude OR of 1.20.

Table 4. Distribution of GSTM1, GSTT1 and GSTP1 genotypes and their association with occurrence of relapse.

\begin{tabular}{ccccc}
\hline GENOTYPE & No Relapse & $\begin{array}{c}\text { Presence of } \\
\text { Relapse }\end{array}$ & OR (95\% CI) & $\begin{array}{c}\text { P } \\
\text { value }\end{array}$ \\
\hline GSTM1 & & & & \\
Present & $6(42.9 \%)$ & $8(57.1 \%)$ & & 0.40 \\
Null & $18(56.3 \%)$ & $14(47.7 \%)$ & $0.58(0.16-2.07)$ & \\
GSTT1 & & & & \\
Present & $12(54.6 \%)$ & $10(45.4 \%)$ & & 0.76 \\
Null & $12(50.0 \%)$ & $12(50.0 \%)$ & $1.20(0.38-3.83)$ & \\
GSTP1 & & & & 0.661 \\
Ile/Ile & $13(54.2 \%)$ & $11(45.8 \%)$ & & \\
Ile/Val & $10(47.6 \%)$ & $11(52.4 \%)$ & $1.3(0.40-4.21)$ & \\
Val/Val & $1(100 \%)$ & $0(0 \%)$ & & \\
\hline
\end{tabular}

Table 5. Crude and adjusted odds ratios for occurrence of adverse events after controlling for sex and age at diagnosis.

\begin{tabular}{lcc}
\hline & Crude OR (95\% CI) & Adjusted OR (95\% CI) \\
\hline GSTM1 & $0.9(0.08-6.54)$ & $1.0(0.14-6.86)$ \\
GSTT1 & $1.6(0.23-11.95)$ & $1.15(0.20-6.69)$ \\
GSTP1 & $0.86(0.15-4.8)$ & $0.85(0.13-5.50)$ \\
\hline
\end{tabular}

Table 6. Crude and adjusted odds ratios for occurrence of relapse after controlling for sex, age at diagnosis, and presence of CNS disease at diagnosis.

\begin{tabular}{lcc}
\hline & Crude OR (95\% CI) & Adjusted OR (95\% CI) \\
\hline GSTM1 & $0.58(0.16-2.07)$ & $0.23(0.05-1.20)$ \\
GSTT1 & $1.20(0.38-3.83)$ & $0.69(0.18-2.64)$ \\
GSTP1 & $1.3(0.40-4.21)$ & $1.21(0.32-4.51)$ \\
\hline
\end{tabular}




\section{Discussion}

Acute lymphoblastic leukemia (ALL) is the most common cancer in children and is one of the most curable of the pediatric malignancies. Many factors have been identified as having prognostic significance in the outcome of patients with ALL. The standard factors are age and WBC at diagnosis, with infants (less than one year), adolescents (greater than nine years), and children with WBC above $50,000 / \mu \mathrm{l}$ being at higher risk. ${ }^{12}$

The promise of pharmacogenetics lies in its potential to identify the right drug and dose for each patient. Even though individual differences in drug response result from the effects of age, sex, disease, or drug interactions, genetic factors also influence both the efficacy of a drug and the likelihood of an adverse reaction. ${ }^{13}$ In an endeavor to develop individualized treatment for acute lymphocytic leukemia using potential genetic markers, we conducted a pilot study investigating the association of GST polymorphisms and treatment response in 46 children afflicted with ALL.

Glutathione-S-transferases (GSTs) are a family of cytosolic enzymes involved in the detoxification of reactive species by catalyzing the conjugation of mutagenic electrophilic substrates to glutathione which is water-soluble and readily excreted from the body. It was shown that differences in the GST enzyme activity mediated by polymorphic genes confer varying susceptibility to environmental cancer as well as response to chemotherapeutic treatment. ${ }^{5}$

Several studies on GST polymorphisms and its association with disease susceptibility and treatment response have been done on different types of cancers, particularly on acute lymphocytic leukemia. However, the results of some studies were found to be contradicting. Haranatha et al. reported a weak association of GSTM1 and GSTP1 polymorphisms with susceptibility to childhood ALL and preliminary study analysis showed improved treatment outcome in patients with double gene deletion. ${ }^{5}$ In contrast, Alcausin et al. reported a trend towards protection from having childhood ALL with presence of the wild GSTT1 and GSTM1 polymorphisms in the Philippines. ${ }^{11}$ Also, Takanashi et al. found that in Japanese patients, the presence of the GST double null genotype was associated with a higher risk of developing early relapse in childhood B-precursor ALL. ${ }^{14}$ In South India, Suneetha et al. showed a significant association between GSTP1 heterozygous and null genotypes and poor outcome. ${ }^{10}$ On the other hand, a study done by Stanulla et al. showed that the GSTP1 null genotype conferred a 3-fold decrease in risk of relapse while the GSTM1 and GSTT1 null genotypes conferred a 2 -fold and 2.8 fold reduction in risk of relapse, respectively. ${ }^{8}$ In a study on gene dose effects involving 263 childhood ALL patients where grouping of GSTM1 and GSTT1 into poor (0 or 1 gene copy) and good metabolizers (at least 2 gene copies) was done, it was shown that the poor metabolizers had a trend toward a better outcome compared with the good metabolizers in terms of event-free survival, and that the good metabolizers demonstrated a 2.2-fold higher risk trend of experiencing resistant disease or relapse compared with the poor metabolizers. ${ }^{16}$

In this study, the adverse events experienced by the study population during treatment included fever, infection, vomiting, elevation of liver enzymes, allergy to LAsparaginase (Leunase), chemotherapy-induced myelosuppression, and death. L-Asparaginase is an important and universal component of therapy for childhood acute lymphoblastic leukemia (ALL). Hypersensitivity reactions to L-Asparaginase occur in up to $30 \%$ of patients and are frequently associated with the development of neutralizing antibodies. ${ }^{15}$ The three most common adverse events were infection, fever, chemotherapy-induced myelosuppresion and death. The presence of GSTT1 null genotype showed a trend towards predisposition to occurrence of adverse events, but this association was weakened when adjusted odds ratios were computed controlling for sex and age at diagnosis. A trend towards predisposition to elevation of liver enzymes during chemotherapy was also observed in patients with GSTT1 and GSTP1 mutant genotypes. In the study of Suneetha et al., it was also shown that the patients harboring GSTP1 heterozygous genotype had lower event-free survival. ${ }^{10}$ Furthermore, the presence of GSTT1 null genotype showed an increased predisposition towards infection during treatment, The presence of GSTM1 null genotype showed a trend towards protection from occurrence of relapse basing on both crude and adjusted odds ratios, similar to the results of the study done by Stanulla. ${ }^{8}$ However, the results obtained in present study are not statistically significant.

It must be noted that the chemotherapy protocols used in the ALL patients varied, namely, the CCG protocol, the Standard High-Risk ALL protocol, and the BFM protocol. One limitation of this study is that due to the small sample size, the analysis of the data was done under one group while it is ideal to analyze the data under three different treatment groups.

\section{Conclusion}

This study shows that certain GST polymorphisms may predispose to infection or elevated liver enzymes during chemotherapeutic treatment of acute lymphoblastic leukemia, however, the results are not statistically significant. Possible associations can be made if a large scale study is undertaken.

\section{Acknowledgment}

The authors gratefully acknowledge Prof. Cynthia Cordero for her invaluable assistance with the statistical analysis. 
References

1. Ma H, Sun H, Sun X. Survival improvement by decade of patients aged 0-14 years with acute lymphoblastic leukemia: a SEER analysis. Sci Rep. 2014 Feb; 4:4227.

2. Huang RS, Ratain MJ. Pharmacogenetics and pharmacogenomics of anticancer agents. CA Cancer J Clin. 2009; 59(1):42-55.

3. Rocha JC, Cheng C, Liu W, et al. Pharmacogenetics of outcome in children with acute lymphoblastic leukemia. Blood. 2005; 105(12):4752-8.

4. Pui CH, Relling MV, Downing JR. Acute Lymphoblastic Leukemia. N Engl J Med. 2004; 350(15):1535-48.

5. Haranatha RP, Kaiser J. Polymorphisms in the GST (M1 and T1) gene and their possible association with susceptibility to childhood acute lymphocytic leukemia in Indian population. Afr J Biotechnol. 2006; 5(16):1454-6.

6. Cho HJ, Lee SY, Ki CS, Kim JW. GSTM1, GSTT1 and GSTP1 Polymorphisms in the Korean population. J Korean Med Sci. 2005; 20(6):1089-92.

7. Rimando MG, Chua MN, Yuson E, de Castro-Bernas G, Okamoto T. Prevalence of GSTT1, GSTM1 and NQO1 $(609 \mathrm{C}>\mathrm{T})$ in Filipino children with ALL (acute lymphoblastic leukaemia). Biosci Rep. 2008; 28(3):11724.

8. Stanulla M, Schrappe M, Brechlin AM, Zimmerman M, Welte K. Polymorphisms within glutathione S-transferase genes (GSTM1, GSTT1, GSTP1) and risk of relapse in childhood B-cell precursor acute lymphoblastic leukemia: a case-control study. Blood. 2000; 95(4):1222-8.

9. Imanishi H, Okamura N, Yagi M, et al. Genetic polymorphisms associated with adverse events and elimination of methotrexate in childhood acute lymphoblastic leukemia and malignant lymphoma. J Hum Genet. 2007; 52(2):166-71.
10. Suneetha K, Nirmala Nancy K, Rajalekshmy KR, Rama R, Sagar TG, Rajkumar T. Role of glutathione-s-transferase and CYP1A1*2A polymorphisms in the therapy outcome of south Indian acute lymphoblastic leukemia patients. Indian J Med Paediatr Oncol. 2011; 32(1): 25-9.

11. Alcausin MMLB, Fajardo PD, Silao CLT, et al. Genetic polymorphisms of Glutathione-S-Transferases P1, T1 and M1 in pediatric patients with acute lymphocytic leukemia in a Philippine Tertiary Hospital. Acta Med Philipp. 2008; 42(2):22-6.

12. Friedmann AM, Weinstein HJ. The Role of Prognostic Features in the Treatment of Childhood Acute Lymphoblastic Leukemia. Oncologist. 2000; 5(4):321-8.

13. Weinshilboum R. Inheritance and drug response. N Engl J Med. 2003; 348(6):529-37.

14. Takanashi M, Morimoto A, Yagi T, et al. Impact of glutathione Stransferase gene deletion on early relapse in childhood B-precursor acute lymphoblastic leukemia. Haematologica. 2003; 88(11):1238-44.

15. Silverman LB, Supko JG, Stevenson KE, et al. Intravenous PEGasparaginase during remission induction in children and adolescents with newly diagnosed acute lymphoblastic leukemia. Blood. 2010; 115(7):1351-3.

16. Borst L., Buchard A., Rosthoj S., et al. Gene Dose Effects of GSTM1, GSTT1 and GSTP1 polymorphisms on outcome in childhood acute lymphoblastic leukemia. J Pediatr HematolOncol.2012; 34(1):38-42.

\section{Acta Medica Pfilippina \\ THE NaTiOnal HeaLTH SCIENCE JOURNAE

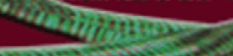

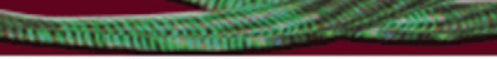 \\ Publishing since 1939}

\section{Acta is now accepting membership}

Privileges of members:

- Allows you to submit articles for possible publication

- Have access to all the articles in the website (archives included) which can be downloaded and printed in pdf format

- Advertise your products/services in the available spaces of the website (for approval of the Editor-in-chief)

For details, please visit our website at www. actamedicaphilippina.com.ph or e-mail us at businessmanager@actamedicaphilippina.com.ph for any questions or queries. 